\title{
El «Arco triunfal a la gloria de Carlos IIl» de Juan Pedro Arnal
}

\author{
Jose LUIS SANCHo *
}

La arquitectura neoclásica española que se realiza cuando Llaguno escribe la primera historia general de nuestra arquitectura, ha sido desde aquel mismo momento objeto de una copiosa bibliografía en la que, no obstante, poco han cambiado los focos de atención y los tópicos. El prestigio convencional de Sabatini como arquitecto - cuando su importancia habría de ser más bien valorada en función de otros criterios, como la influencia sobre la ordenación del territorio español a través del cuerpo de ingenieros militares-, las ensalzadas figuras de Ventura Rodríguez y Juan de Villanueva, han dejado siempre en segundo plano a otras personalidades, o a aspectos más generales de la problemática arquitectónica hasta la reciente aparición del libro del profesor Sambricio sobre La arquitectura española de la llustración ${ }^{1}$, donde se trata por vez primera un tema tan interesante como la influencia de la teoría arquitectónica francesa en un medio como el español dominado por el modo italiano.

En este contexto adquiere singular relieve, como bien ha destacado Sambricio, la figura de Pedro Arnal como campeón de las teorías francesas en la Academia de San Fernando, órgano a través del cual el Estado absolutista intentó imponer una imagen arquitectónica al país sirviéndose, en concreto, de la Comisión de arquitectura a cuya cabeza se encontraba el mismo Arnal. Sin embargo no ha de entenderse la personalidad de este arquitecto, que diseño mucho más que construyó, de una

\footnotetext{
* En la Biblioteca del Palacio Real de Madrid.

Madrid, Instituto de Estudios de Administración Local y Consejo Superior de los Colegios de Arquitectos de España, 1986.
} 
forma simplista, pues tanto en la teoría como en la práctica dejó en muchas ocasiones los modelos franceses por los italianos, y de hecho nunca fue más francés que en sus primeros diseños conocidos, existentes en la Biblioteca del Palacio Real de Madrid y que aquí reproducimos: el "Arco de triunfo a la gloria de la feliz entrada y acceso a la corona de España del rey don Carlos III’².

\section{JUAN PEDRO ARNAL EN EL NEOCLASICISMO ESPAÑOL}

La trayectoria de Arnal y sus realizaciones arquitectónicas, así como su trascendencia en el marco de la Academia, han sido estudiadas en detalle por Sambricio pero aquí interesa destacar algunos aspectos de su personalidad $^{3}$. Aunque Bourgoing le considerase francés, Juan Pedro Arnal había nacido en Madrid en 1735, hijo de un platero francés que le envió a estudiar a la Academia de Toulouse ${ }^{4}$, cuya importancia para la

2 Biblioteca del Palacio Real, Dib. roll. 1, 1-4. Los dibujos han figurado en la reciente exposición "Carlos III, alcalde de Madrid", patrocinada por el Ayuntamiento de la Villa, pero no estaban reproducidos como correspondía en el catálogo junto a su ficha, redactada por quien esto escribe.

3 Sambricio, obra citada, pág. 304-310, y 93-108 (“Juan Pedro Arnal y la teoría arquitectónica en la Academia de San Fernando de Madrid», publicado antes en Goya, núm. 147, nov. 1978, pág. 147-157. Véase además R. MESURET, "Documents et références sur Pierre Arnal, élève de l'Academie Royale de Toulouse et architecte de la maison d'Albe", Caravelle, 1966, pág. 73-81. C. SAmBricio, "Juan Pedro Arnal, arquitecto del siglo Xvill", Archivo Español de Arte, 183, pág. 299-308. La bibliografía no citada aquí puede verse en los artículos de Sambricio.

Ya redactado este texto ha aparecido el libro coordinado por Carlos Sambricio La Casa de Correos, un edificio en la ciudad (Comunidad de Madrid, Consejería de Política Territorial, Madrid 1989) en uno de cuyos capítulos la profesora doña Virginia Tovar realiza nuevas aportaciones documentales a la figura de Arnal, especialmente sobre su actividad en el campo de la arquitectura doméstica. Por desgracia no constrasta los planos del Archivo de Villa con la realización cuando ésta se ha conservado, como la casa de Pérez de Lema en la calle de las Aguas. Sigue a Mesuret en cuanto a la etapa tolosana de Arnal.

4 E. Llaguno y J. A. Cean Bermúdez, Noticia de los arquitectos y arquitectura en España, Madrid 1829, IV, pág. 308: “Uno de los arquitectos más eruditos de su tiempo. Nació en Madrid el día 19 de Noviembre de 1735, y el siguiente fue bautizado en la iglesia de San Luis... Su padre Juan Enrique Arnal, natural de Perpiñan y platero acreditado en esta corte, y su madre Margarita Gerónima Ardei, natural de Castelnodari, diócesis de Sanpapul en Francia, que vivian en la calle de la Montera, le dieron una decente educación, y le enviaron a Tolosa de aquel reino a estudiar las bellas artes, en cuya Academia obtuvo siete premios en la arquitectura, en la perspectiva y en el dibujo". 
penetración en España de las nuevas tendencias francesas ha sido destacada por Mesuret y Sambricio. Alli estuvo de 1758 a 1762 formándose en el gusto clásico francés, de lo que son buena prueba estos diseños de Arco triunfal y Fuente, presentados por Arnal en 1758 y 1759 a los concursos de aquella academia.

De Toulouse trajo un bagaje teórico considerable así como un gusto arquitectónico basado en la tradición clásica francesa y orientado hacia los nuevos problemas compositivos que recién llegado a Madrid en 1763 le harian entrar en abierta contradicción con la Academia de San Fernando, ajena a una y a otros, lo cual explica la mala fortuna del alumno en sus primeros proyectos presentados a la Academia madrileña en ese año y que recibieron una crítica severa por arquitectos españoles como Diego de Villanueva, Hermosilla y Castañeda.

La educación tolosana del joven Arnal se concretó en dos rasgos ya subrayados por Llaguno y que nos definen su personalidad: una dedicación como teórico predominante sobre su actividad arquitectónica, y una tendencia al diseño y realización de obras de "ornato".

En palabras de Llaguno-Ceán, «Fue uno de los profesores más eruditos e instruidos de estos tiempos, pues además de poseer las partes científica y práctica de la arquitectura, estaba muy versado en su historia, y en la de las demás bellas artes con la frecuente lectura de libros facultativos y biográficos que conservaba, pues se había propuesto comprar cuantos se publicaban en Francia de este género" ${ }^{5}$. Siendo director general de la ilustre Casa en 1801-1804, "regaló a la misma Academia nueve obras artísticas, impresas casi todas en idioma francés, y adornadas con estampas, de que carecía, para colocarlas en su biblioteca pública». Como ha señalado Sambricio, las ideas que se filtran por este conducto no se limitan a lo arquitectónico sino que forman parte de la utopía social de la llustración, y de este modo cabe valorar la importancia de la Academia de Toulouse en nuestro país mayor en los campos de las matemáticas o del pensamiento que en el arte específico de construir. "Gracias a figuras como la de Arnal o Bails se produce la paradoja de que la Academia consiga acceder a algunos estudios generales no arquitectónicos antes de que los reformadores sociales fijen su atención en éstos" ${ }^{\circ}$.

5 Ibidem,, pág. 309.

6 Sambricio, La arquiectura española..., pág. 98. 
Sin embargo este peso en la teoría académica y en la formación de los arquitectos de la tercera generación ilustrada no lo ejerce Arnal mediante la redacción de textos, sino a través de comentarios verbales de un indiscutido valor y mediante su puesto como presidente de la Comisión de arquitectura de la Academia, que cestablecida en $1786 \ldots$ tenía como misión vigilar y censurar la totalidad de las obras públicas que se realizaban en España". Se crea de este modo una red de profesionales que definen la imagen arquitectónica de la España llustrada según se configura en el reinado de Carlos IV, aspecto poco atendido dada su importancia, también destacado por Sambricio ${ }^{7}$. Sin embargo es preciso señalar que el peso de la teoría incide más en lo relativo a la problemática general de la composición que en aspectos formales, en los cuales el propio Arnal se aparta cada vez más del modelo francés para adaptarse a los tipos italianizantes en uso en el Madrid carolino. Así, cuando en 1795 diseña la fachada de la Imprenta Real en la calle de Carretas no puede evitar la vuelta al esquema de palacio romano que treinta años antes habia consagrado Sabatini en la Aduana, bien por imposición del genius loci de la Corte o por decidida regresión. Aún más problemática a este respecto es su intervención en el palacio de Buenavista, donde siguiendo a Llaguno sólo pueden atribuírsele «la portada y reparos», quedando el resto, es decir la composición general que es precisamente lo más avanzado, al enigmático arquitecto francés Claude Billiard de Bellisard, cuyo carácter "revolucionario" patente en la maqueta antaño propiedad de los duques de Sueca apenas si es hoy reconocible en el edificio.

Arnal se presenta, a mi juicio, como un arquitecto dividido, "esquizoide", que si por un lado ejerció una valiosa tarea teórica y de difusión de un nuevo rigor compositivo en la Academia, como contrapartida dirigió su inventiva, frustrada en cuanto a realización por la hostilidad del gusto madrileño vuelto hacia Italia, al ornato arquitectónico. Esta es una tendencia ya evidente en este proyecto de arco y que fue impulsada durante su etapa de formación tolosana por su maestro Labal de Savignac, hombre según Mesuret especialmente inclinado al diseño de motivos decorativos aislados según un gusto rococó que merecería la reprobación de Llaguno in testa discipuli: "Tuvo (Arnal) también particular gusto en diseñar, y aun grabar al aguafuerte adornos arquitectónicos, urnas, mausoleos, jarros y muebles, bien que con el que habia adquirido en Tolosa en sus primeros años, no muy conforme al grave y sencillo de nuestros

Ibidem, pág. 100-101. 
antiguos y buenos profesores". Realmente Arnal se apartó en composición del rococó de su maestro, como demuestran los dibujos aqui presentados, pero el párrafo de Llaguno tiene interés por indicar las fuentes francesas, no herrerianas, de su lenguaje, y su concepción de elementos aislados, con lo que no nos referimos sólo a sus fantasías sino también a su obra que mayoritariamente se compone de "ornatos": efímeros - para la Academia, 1789-, portadas - palacio de Buenavista, Madrid; palacio del Viso del Marqués; hospital de Tavera, Toledo-, retablos, tabernáculos, urnas, mausoleos y obeliscos ${ }^{8}$. El juicio de Llaguno-Ceán no sólo indica esta tendencia a considerar la arquitectura como ornato de superficie, sino que revela el carácter exótico que el estilo de Arnal nunca dejó de tener entre la arquitectura española, separada por un abismo de la francesa. Quizá por ello Arnal se conformó con las posibilidades que el medio madrileño le brindaba, encauzando su ingenio por las vías de la teoría y de las pequeñas realizaciones en las que se toleraba o resultaba deseable como adorno una disonancia estilistica.

El empleo a gran escala del lenguaje clasicista en sus proyectos - Arnal es el primer arquitecto español que parece obsesionado por el empleo masivo de columnas y por el tema del obelisco, difundidos luego por Villanueva y su escuela - como en el proyecto para palacio episcopal de 1763, no debe hacer olvidar el carácter a menudo teatral de tales elementos, tan epidérmicos respecto al edificio en Tavera o El Viso como en las diversas variantes propuestas para el ornato de 1789 en la Academia, en uno de los cuales vuelve sorprendentemente a esquemas barroco-clasicistas no ya franceses sino juvarianos, con un ático sobre una columnata gigante, lo cual constituye una cabriola regresiva sorprendente en un arquitecto pretendidamente "revolucionario". Sin embargo Arnal era capaz de concebir un complejo arquitectónico de modo coherente según las tendencias francesas avanzadas, como en su citado proyecto de 1763, que quizá por ello resultó significativamente incomprendido por Castañeda, Hermosilla y Villanueva.

Con estos dos últimos Arnal colaboró en una de las empresas más curiosas de la erudición ilustrada española como es el estudio de las antigüedades árabes de la Alhambra, dedicación arqueológica a la que continuó, por encargo real, en las excavaciones de Rielves, cerca de Toledo ${ }^{9}$. A este respecto nos parece digna de ser destacada la imper-

a Sobre las obras de Arnal cfr. Llaguno-CeAn, obra citada, pág. 310, y SamBricio. La arquitectura española..., pág. 304 y ss.

9 “En 1780 le nombró el rey para que fuese a examinar el descubrimiento de los mosaicos de Rielves cerca de Toledo. Evacuadas las excavaciones del terreno, levantó el 
meabilidad tanto de Arnal como del primer arquitecto español del momento, Juan de Villanueva, respecto al empleo en obras decorativas del repertorio arabesco nazarí, cuando el eclecticismo de finales del XVIII desplegaba ornatos exóticos de variada procedencia en los interiores franceses e ingleses. Cuando ya un autor tan divulgado como Chambers había propuesto pabellones de jardín estilo "Alhambra", cuando Dugourc diseñaba para Carlos IV interiores en los que se lleva al delirio el empleo de lenguajes lejanos en el tiempo y en el espacio, esta omisión por parte tanto de los regios comitentes como del arquitecto induce a variadas reflexiones sobre la relación entre tales erudiciones arquitectónicas y el foco creativo de la Corte; Villanueva y Arnal no parecen haber pisado jamás Granada, fieles a su formación clasicista, romana el uno, francesa el otro.

\section{LOS DIBUJOS DE ARNAL EN LA BIBLIOTECA DE PALACIO}

A este respecto los dibujos de la Biblioteca del Palacio Real no pueden ser más representativos de las ideas estéticas que Arnal recibió en Toulouse, pues se trata de ejercicios académicos de un alumno de veinticuatro años que aplicadamente se inspira en los edificios del clasicismo francés propuestos como modelos. Son, por otra parte, los dibujos más antiguos de Arnal identificados hasta la fecha, y nos muestran ya a un dibujante enormemente hábil no sólo en la parte arquitectónica, sino en la ornamentación y las figuras. Seguramente por ellos obtuvo alguno de los "siete premios de la arquitectura, en la perspectiva y en el dibujo» que según Llaguno obtuvo en Toulouse, y quizás por alguno de ellos el "Grand Prix» que menciona Mesuret.

Los diseños están ejecutados sobre papel pegado en tela, formando cuatro rollos de diversas dimensiones, y corresponden a dos proyectos bien diferenciados, cada uno de ellos con dos planos: la planta en uno de ellos, los alzados y secciones en el otro.

plano del edificio antiguo, e hizo quince diseños de otros tantos pavimentos mosaicos que se encontraron, los que se grabaron después, y existen para su venta en la real calcografía”. 
El arco presenta una composición singular: el volumen cúbico del cuerpo central trae a la memoria la tendencia a esta forma geométrica que aparece en la arquitectura avanzada inglesa y francesa de la llustración. La planta, el alzado y la sección transversal así como las leyendas elocuentes para dar idea de la estructura - según la usanza francesa los entablamentos hechos a base de dovelas se aseguraban con barras de hierro- y de la riqueza iconográfica con que Arnal convertía el Arco en un discurso político:

1. “Plan d'un arc de triomphe à la gloire de l'heureuse entrée et avenement du roy don Carlos III à la Couronne d'Espagne / ouvrage dedié a Sa Majêté Catholique / par son trés humble et trés fidèle serviteur et sujet, Jean Pierre Arnal / Coté de la ville / coté de la principale entrée / Jaun Pedro Arnal natural de Madrid inventado y dibujado». Escala de módulos y de treinta toesas.

2. "Arc de triomphe... elevation de la principale entrée de l'arc de triomphe. Coupe et profil prise sur la ligne A-B. Profil de l'entablement et chapiteau tiré de l'ordre corinthien du Pantheon de Rome. Bosse atique tire de l'ordre ionique du theatre de Marcellus à Rome".

Escala y firmas idénticas al anterior.

Leyenda explicativa de los temas indicados con números en los alzados: 1.-SA MAJESTE. 2.-La Sicile. 3.-L'Espagne. 4.-Les quatre parties du Monde... 5.- La Religion. 6.-La Force. 7.- La Justice. 8.-La Clemence. 9.-Découverte de la ville d'Herculaneum. 10.-Mars personifié par Sa Majesté entouré par des dances el des jeux que les sciences et les arts font en alégresse par la protecttion qu'il leur accorde. 11.-L'amour du peuple. 12.-L'abondance et la richesse par les sages rois. 13.- La fidelité du peuple. 14.-La gloire. 15.-Sujets d'histoire de Sa Majesté. 16.--Bas-relief representant l'hommage rendu au roy par toute l'Espagne "barres de fer servant a soutenir la grande travée de l'entablement liée ensemble par des clefs de fer".

La fuente, asociada con el arco por su estrecha afinidad formal y por tratarse igualmente de un elemento urbanistico tipico de la gran arquitectura de las monarquias absolutas, no guarda sin embargo relación directa con el rey: obviamente le ha sido enviada junto con el Arco por el hecho de ser el proyecto mas reciente del artista.

3. "Plan d'une fontaine publique à la grecque ouvrage dedié a Sa Majesté Don Carlos III roy d'Espagne par...”

Escala en módulos y toesas.

4. «Fontaine publique à la grecque... elevation principale... elevation d'une des façades laterales... coupe et profil sur la ligne A-B".

Leyenda con indicaciones sobre los depósitos y los detalles arquitectónicos: “Profil de l'ordre tiré de celui du temple de la Fortune Virile à Rome. Chapiteau angulaire conforme à celui du temple d'Erechtée a Athene, et à celui du temple de la Fortune Virile à Rome. Basse atique, tiré de l'ordre ionique du theatre de Marcellus, à Rome». 
No se trata, por tanto, de proyectos concebidos para ser realizados, ni mucho menos de encargos, sino de un trabajo académico con la carga de utopía, de expresión de ideales plásticos que ello supone. Los temas son característicos de las Academias de mediados del XVIII, esencialmente ligados a la representación de la magnificencia del poder: hacia finales del siglo cambiará la temática proponiéndose hospitales, colegios, gimnasios, cementerios, lazaretos y otros edificios de utilidad pública. Pero hacia 1750-1760 la gran arquitectura áulica del barroco tardío continuaba dominando las Academias. Ha de señalarse que la de San Fernando emplazó en 1757 como tema para la prueba de repente de la primera clase "un arco de triunfo al modo de los antiguos romanos", y en 1760, para la prueba de pensado de la segunda clase, "Un arco triunfal de cincuenta pies de alto y ochenta de ancho, poco más o menos, dedicado al rey. Planta, elevación y corte, todo geométrico" ${ }^{10}$. Años después, en 1772 , el tema para la de repente de primera clase era una "fuente adornada de arquitectura y escultura en un arco de triunfo que haga frente a la principal calle de un gran jardín". Los proyectos de Arnal estaban, por tanto, plenamente acordes con su tiempo e incluso es posible que presentase su arco en 1760 al concurso de Madrid. La oportunidad del tema es eminentemente política y ninguno de los concursos académicos se hacía pensando en posibilidad alguna de realización, pero no hay que olvidar que por aquellos años comienza a gestarse el proyecto de transformación del Paseo del Prado a manera de "Foro", incorporando fuentes monumentales - bien distintas a la concebida por Arnal- y una entrada monumental a la Villa, la puerta de Alcalá, que superase con mucho a las ya existentes, alguna de ellas edificada hacía poco tiempo, como la de Recoletos: "Aquí hubo el pensamiento, según se dijo, de que la Puerta de Alcalá se ejecutase bajo la idea de un "arco triunfal", y como un monumento del feliz arribo de S. M. a esta Corte. En esta inteligencia se puede considerar por sí sola, y sin respecto a otras fábricas que estén cerca, o lejos de ella».

Con estas palabras comenta Antonio Ponz la relación con su entorno del objeto que ha llegado a ser más representativo de la arquitectura de Carlos III. La Puerta de Alcalá hereda así las funciones de glorificación del poder que en España venían siendo dominio exclusivo de la arquitectura efímera y contribuye a configurar la nueva imagen de Madrid como capital de una monarquía católica ilustrada según los parámetros euro-

10 A. Quintana Martínez, La arquitectura y los arquitectos en la Academia de Bellas Artes de San Fernando, 1744-1774, Madrid 1983, pág. 126-127. Esta autora resume asimismo la trayectoria académica de Arnal hasta 1774. 
peos, al uso de Francia o Italia. La elección del lenguaje arquitectónico será sin embargo inequivocamente italiana por parte de Carlos III y de la Corte mediante la actividad de un grupo de arquitectos napolitanos cuyo jefe y cabeza visible es Sabatini, y sus puntos de referencia la gran tradición moderna italiana cuyo canto de cisne habian entonado Fuga y Vanvitelli. Así Sabatini emplea en la Puerta de Alcalá «los capiteles... que inventó el gran Miguel Ángel para la fábrica del Capitolio en Roma, en cuya ciudad se hicieron modelar, con el fin de usar de ellos en esta Puerta. Se ha logrado con eso, sin ir a Roma, que se vean en Madrid puestos en obra dichos capiteles. Ventaja sería, que así como se copian cuadros o estatuas se copiaran también los edificios más insignes de aquella ciudad, o de otros parajes" ".

El mérito indiscutible de Sabatini, lo que le daba el respaldo del rey y de la Corte, era la elección del modelo, el romano antiguo y moderno, por parte del comitente. Arnal diseña conforme a la misma teoria, propia del clasicismo barroco, de la copia o imitación de los buenos ejemplos antiguos, pero en su caso se trata del vocabulario clásico francés cuya utilización ha señalado Sambricio en el proyecto Arnal de 1763 para la Academia. De modo aún más evidente el mismo espíritu anima este ejercicio del joven alumno de la de Toulouse que, inspirándose de modo directo en la ordenación de la columnata del Louvre de Perrault, demuestra su maestría en el manejo de elementos tomados de la arquitectura griega y romana fielmente utilizados como cita erudita más que como modelos. La fuente, que de griega apenas tiene algo más que el capitel tomado del Erecteion, es en cierto modo otra versión más pequeña de fachada monumental ordenada por pares de columnas a manera de un arco de triunfo, reelaborando en una clave completamente diversa los modelos más prestigiosos de este género específico como el Acqua Paola de Roma (1610). Una comparación de este alzado con el propuesto por Ventura Rodríguez para la fuente del palacio de Boadiola o el primer proyecto de la puerta de Alcalá revela hasta qué punto podían diferir sobre un mismo tema los lenguajes no entendidos por Carlos III. Aún más elocuente a este respecto es la comparación con los proyectos de Sabatini para la puerta de Alcalá (Gijón, col. Marin Rodriguez de Rivas) que podía fácilmente hacerse en la exposición citada, y con los de Ventura Rodríguez con el mismo objeto: los tres son lenguajes del barroco tardío, pero mientras Arnal se expresa en francés, los otros dos son dialectos diversos del italiano: la elección del estilo del rey no fue a favor

11 A. Ponz, Viaje de España, tomo V, pág. 7-8 (edición de 1793). 
del más avanzado, sino del que le ofrecía un modelo y un marco de referencias acorde con los ideales de la monarquía.

Arnal ofrece en estos diseños las bases de referencia sobre las que podía haberse elaborado una arquitectura neoclásica española completamente distinta pero que, falta de salida, quedó anquilosada como lo demuestran los proyectos para el efímero de la Academia en 1789; una vía, la suya, que quedó limitada a la especulación teórica y a la arquitectura ornamental. 
El «arco triunfal a la gloria de Carlos III»...

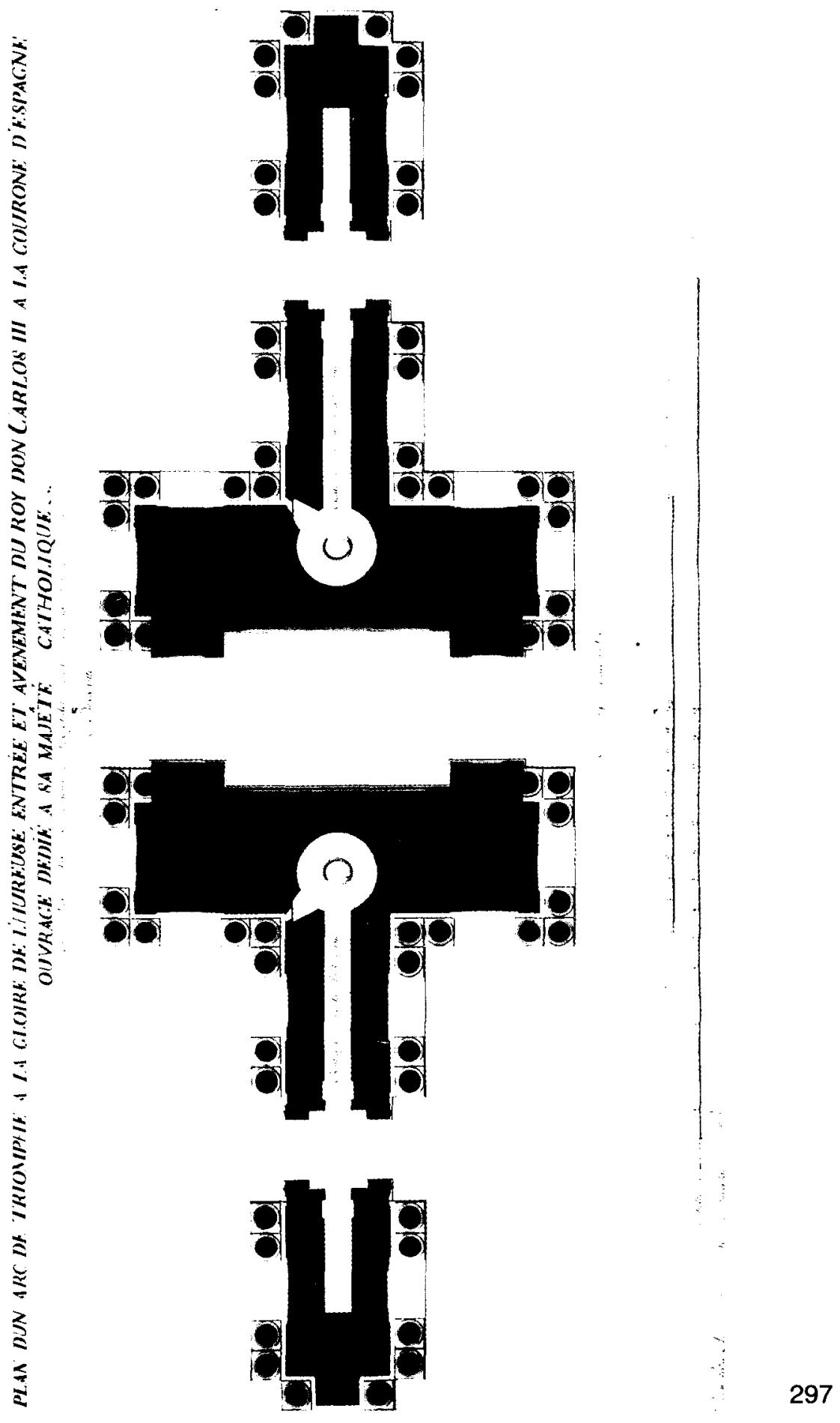




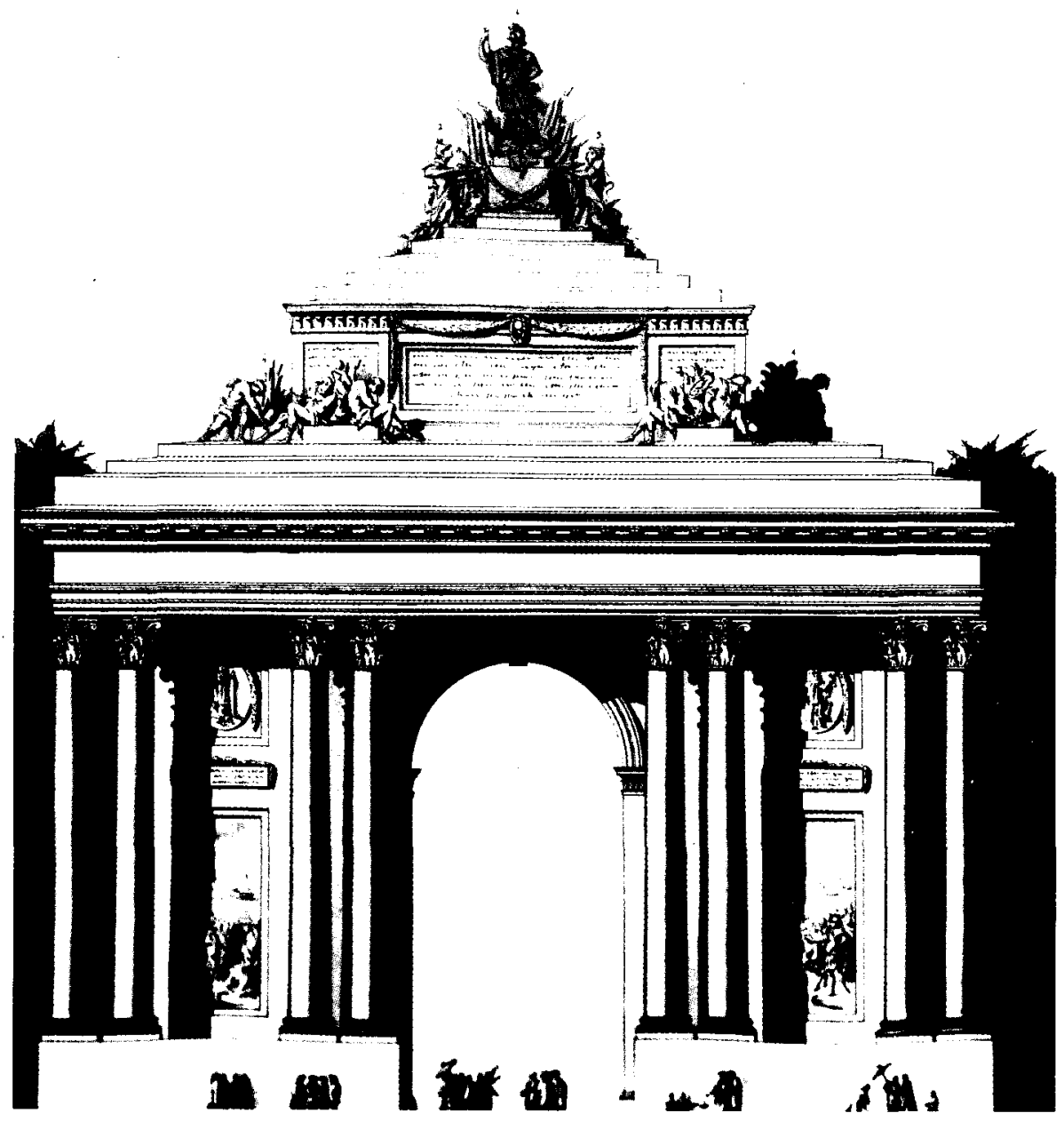


El «arco triunfal a la gloria de Carlos III»...

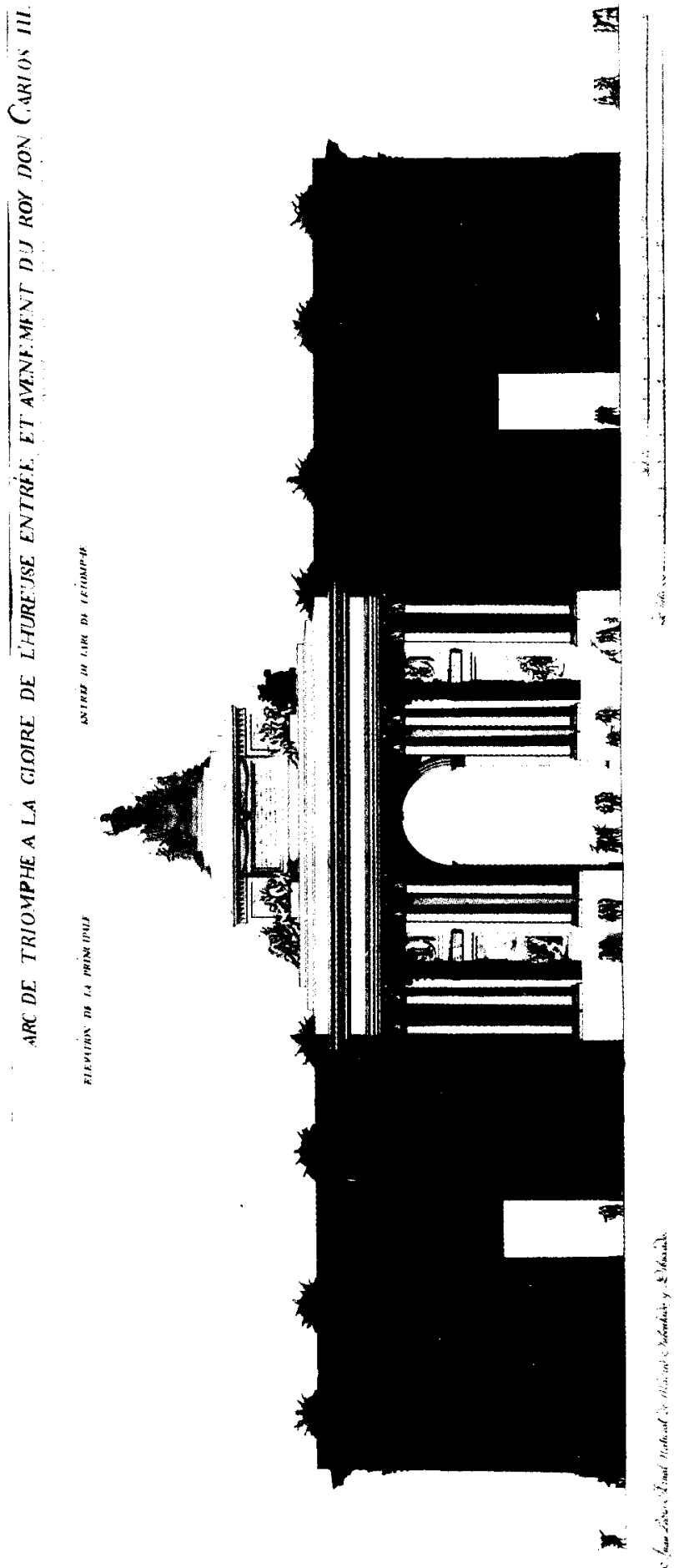




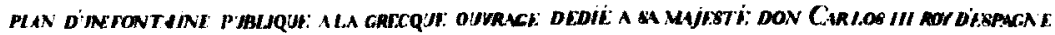

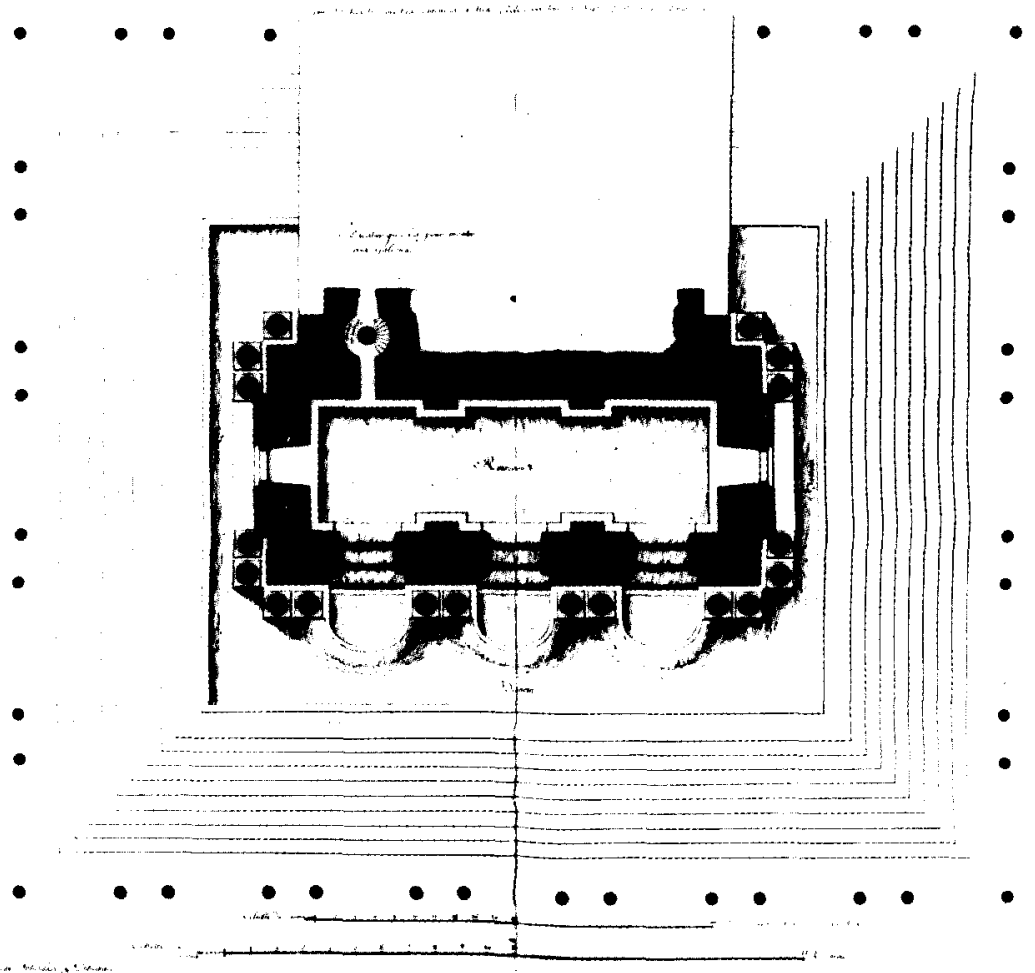




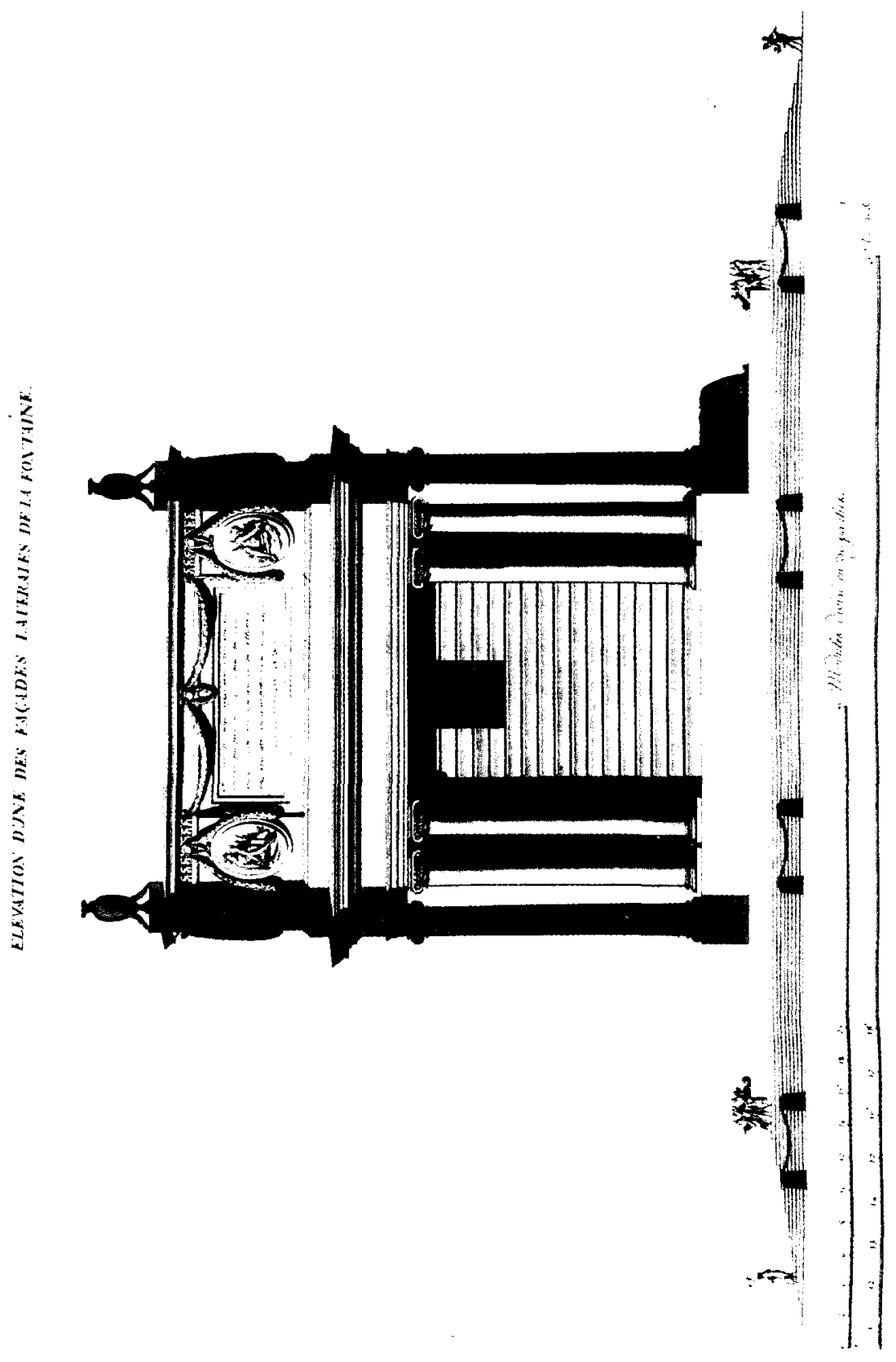




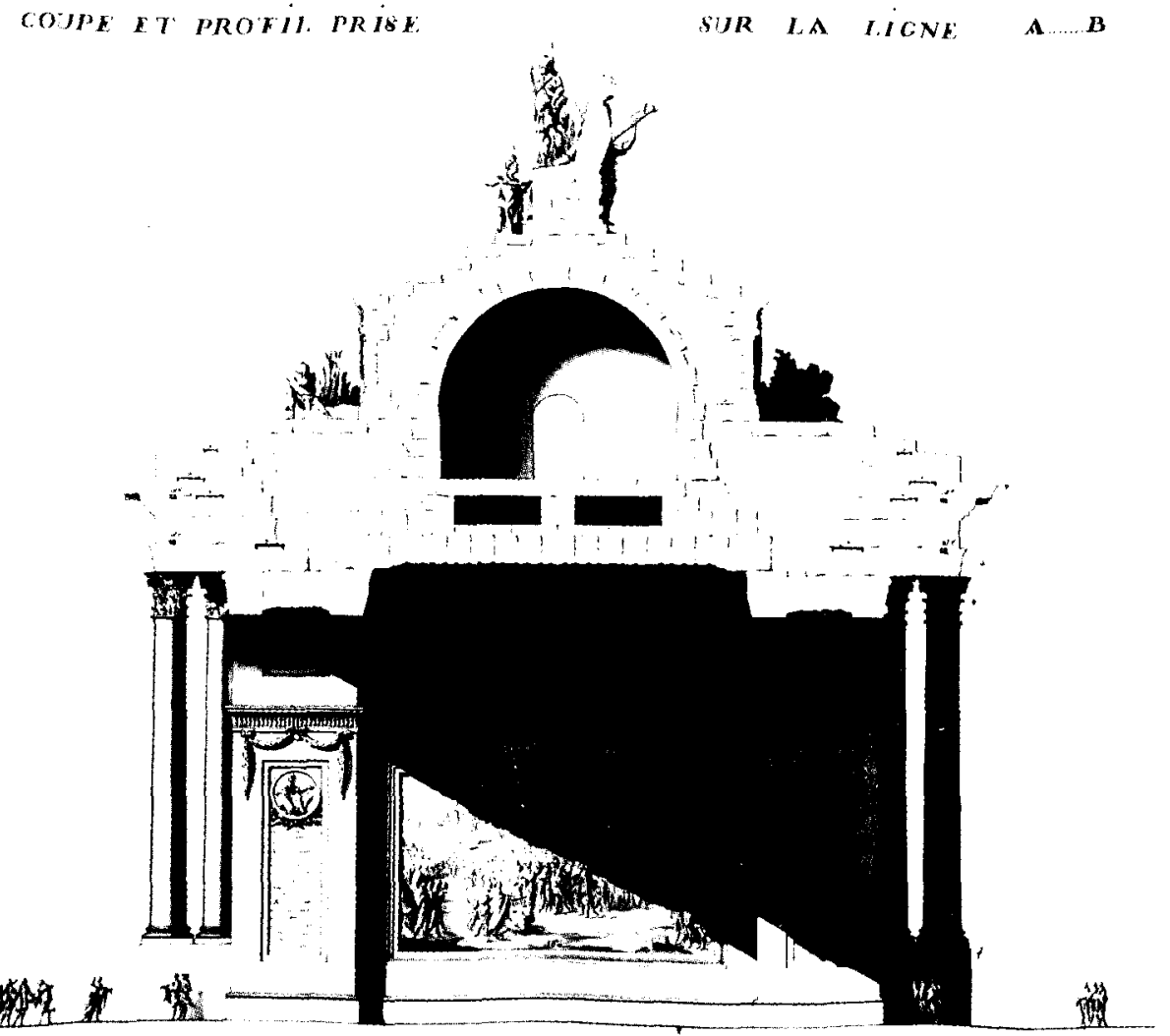

